\title{
Stability and rheological properties of corn oil and butter oil emulsions stabilized with texturized whey proteins by supercritical fluid extrusion
}

\begin{abstract}
Texturized whey protein powder (tWPC) produced by supercritical fluid extrusion process was used to stabilize butter and corn oil emulsions. The stability of emulsions prepared at various oil concentrations and storage temperatures was investigated. Emulsion morphology was observed by CLSM and quantified for size distribution of oil droplets while rheological properties were examined to establish their stability. All corn oil-based emulsions were stable against droplet coalescence during storage. However, the stability of butter oil-based emulsions was significantly influenced by storage temperatures and oil concentrations. Emulsions with higher elasticity were obtained by raising the oil concentration. Corn oil emulsions were far less sensitive to variations in storage temperature than emulsions based on butter oil. tWPC have a potential for uses in a very large set of food emulsions from low to high fat content and from Newtonian consistency to elastic, solid like materials such as salad dressings, light mayonnaises, fillings, sandwich spread and spreadable butter.
\end{abstract}

Keyword: Texturized whey protein concentrate; Oil-in-water emulsion; Stability; Droplet coalescence; Rheological properties 